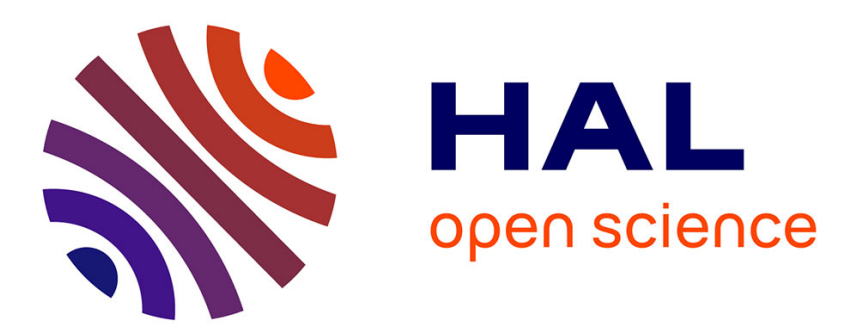

\title{
On the Influence of Medium Statistics on the Robustness of Time-Reversal Transmissions
}

\author{
Andrea Cozza, Florian Monsef
}

\section{To cite this version:}

Andrea Cozza, Florian Monsef. On the Influence of Medium Statistics on the Robustness of TimeReversal Transmissions. IEEE Antennas and Wireless Propagation Letters, 2016, 16, pp.38-41. 10.1109/LAWP.2016.2553148 . hal-01299198

\section{HAL Id: hal-01299198 \\ https://hal-centralesupelec.archives-ouvertes.fr/hal-01299198}

Submitted on 7 Apr 2016

HAL is a multi-disciplinary open access archive for the deposit and dissemination of scientific research documents, whether they are published or not. The documents may come from teaching and research institutions in France or abroad, or from public or private research centers.
L'archive ouverte pluridisciplinaire HAL, est destinée au dépôt et à la diffusion de documents scientifiques de niveau recherche, publiés ou non, émanant des établissements d'enseignement et de recherche français ou étrangers, des laboratoires publics ou privés. 


\title{
On the Influence of Medium Statistics on the Robustness of Time-Reversal Transmissions
}

\author{
Andrea Cozza, Senior Member, IEEE, Florian Monsef, Member, IEEE
}

\begin{abstract}
The problem of predicting the performance of TimeReversal Transmissions (TRT) in a time-varying complex medium is addressed in this paper. The loss of coherence in the propagation medium and its nominal energy contrast are proven sufficient to predict the average loss of quality in received signals. In particular, it is shown how a perturbation in the medium affects in a different way coherent focusing and background fluctuations. In the extreme case of a diffusive medium (e.g., Rayleigh channels), the fluctuations intensity is unaltered. The predictions of the proposed theoretical models are validated against experimental results measured in a reverberation chamber, where a mechanical paddle (stirrer) acts as the source of perturbation in the medium. It is confirmed that, depending on the medium statistics, perturbations can have a varying impact on TRT performance.
\end{abstract}

Index Terms-Time-reversal transmissions, complex media, perturbation analysis.

\section{INTRODUCTION}

Waves propagating through complex media are subjected to a potentially large number of scattering events, generating multiple propagation paths and ultimately leading to phenomena like time-spreading of pulses and frequency selectivity, making signal transmissions less predictable and reliable.

Time-reversal transmissions (TRT) have been studied in the last few years as a potential solution to counter these effects, by providing the best replica of a target signal at the receiver, while at the same time focusing a substantial portion of the energy around it [1], [2]. Most applications are based on a direct implementation of standard time reversal as described in [3]. In short, in order to have a received signal as close as possible to a target signal $p(t)$, the transmitted signal has a Fourier spectrum of the kind $P(\nu) H^{*}(\nu) M(\nu)$, where $\nu$ is the frequency variable and $H(\nu)$ is the transfer function between the transmitter and the receiver. $M(\nu)$ is a weighting function that represents the effects of equalization and compensation schemes that have been used for improving TRT [4].

The main weakness of TRT is that even in collaborative configurations, where it makes sense to assume $H(\nu)$ to be known, propagation media may change over time. As a matter of fact, real-life propagation scenarios are hardly static, as they often involve moving people/vehicles, and also receiving and transmitting antennas can change position/orientation, e.g., when hand-held mobile terminals are used. Time-varying propagation can be taken into account, by periodically sounding the

Part of the work here reported was funded by the French National Research Agency through the grant ANR-12-ASTR-0005, MIMOCHIC project, within the framework of the 2012 ASTRID program.

A. Cozza and F. Monsef are with PIEM, GeePs (UMR 8507), 11 rue Joliot-Curie, 91192 Gif-sur-Yvette, France. Contact e-mail: andrea.cozza@ieee.org. medium response and transfer functions, but this can clearly be done only at discrete times. In between them, TRT risk using signals that no longer represent an accurate description of the medium, resulting in a loss of coherence and ultimately a reduced performance. This problem has been addressed in a few papers, mostly empirically and essentially with the aim of assessing what degree of perturbation would still provide an acceptable performance [5]-[8]. In diffusive media, such as large cavities, indoor propagation and heavily built urban structures, TRT can be so sensitive that it has been proposed for detecting perturbations in the first place [9].

A case therefore exists to understand under what conditions TRT are more of less sensitive to perturbations. This paper brings some insight into this issue, by demonstrating how the statistical behavior of a medium results into a different sensitivity to perturbations. The predictions of the proposed models are validated against experimental results, confirming their accuracy.

\section{RESIDUAL FLUCTUATIONS AND CONTRAST}

Signals received with TRT in static media can be expressed as

$$
y(t)=\int_{B_{T}} \mathrm{~d} \nu P(\nu)|H(\nu)|^{2} \mathrm{e}^{\mathrm{j} 2 \pi \nu t}
$$

where $H(\nu)$ is the transfer function between the transmitting antenna and the receiver and $B_{T}$ is the bandwidth over which the spectrum $P(\nu)$ of the target signal $p(t)$ is defined. For any medium significantly different from free space, $H(\nu)$ would not be a monotonous function of frequency, presenting minima or even nulls over certain frequencies due to reflections and in general interactions with boundary elements. Modelling $H(\nu)$ as a random process, fluctuations in $y(t)$ appear as the result of deviations from line-of-sight transmissions.

The effectiveness of TRT can be measured by first separating the received signal $y(t)$ as done in [10]

$$
y(t)=\alpha p(t)+f(t),
$$

i.e., as made up of a coherent portion proportional to $p(t)$ and residual fluctuations $f(t)$. Projecting the received signal onto the target one yields

$$
\alpha=\langle Y(\nu), P(\nu)\rangle=\int_{B_{T}} \mathrm{~d} \nu Y(\nu) P^{*}(\nu),
$$

choosing $p(t)$ to have unit energy, here meant as for a mathematical signal, i.e., the square of its $L_{2}$ norm. A direct consequence of (2) is the orthogonality of $f(t)$ and $p(t)$.

A natural metric is therefore the energy contrast $\Lambda$ [10], which measures the ratio between the energy $\mathscr{E}_{C}=\alpha^{2}$ of the 
portion of received signal proportional to the target $p(t)$ and that of the background fluctuations, $\mathscr{E}_{f}$, i.e.,

$$
\Lambda=\mathscr{E}_{c} / \mathscr{E}_{f} .
$$

Sec. III is entirely based on this metric, which strongly simplifies the analysis.

For a diffusive medium, it was shown [10] that its ensemble average $\langle\Lambda\rangle \leq 1$, with the extreme case attained only in the case of a Rayleigh or diffusive medium. Introducing the TRT function $W(\nu)=|H(\nu)|^{2}$, [10] demonstrated that the second-order statistics of $W(\nu)$ provide a direct estimate of the energy contrast as $\langle\Lambda\rangle=\varsigma_{W}^{-2}$, where $\varsigma$ is the standard deviation of $W(\nu)$ normalized to its mean. This relationship is useful as it gives a straightforward understanding of the performance of TRT as a function of the medium statistics and complexity. Nevertheless, it only holds for the average behavior of a medium, so that $\Lambda$ can exceed the value one on a local basis, as observed in Sec. IV.

Once the energy contrast is known, it can be translated into a peak contrast, measuring the ratio of the peak of coherent focusing and the rms amplitude of the background fluctuations [10].

\section{EFFECTS OF PERTURBATIONS ON TRT}

We consider a nominal transfer function $H_{o}(\nu)$ for the unperturbed medium and $H(\nu)$ the one found at the time of transmission, after the perturbation event has occurred; in general, all quantities referring to the unperturbed medium will display a subscript nought. It is convenient to express the modified transfer function as a linear combination of the original function plus a random process $\delta H(\nu)$ modelling the effect of the perturbation, i.e.,

$$
H(\nu)=\rho H_{o}(\nu)+\delta H(\nu),
$$

with $\rho$ the linear correlation coefficient between the original and modified functions. By definition, $\delta H(\nu)$ is orthogonal to $H_{o}(\nu)$, i.e., their projection

$$
\left\langle\delta H(\nu), H_{o}(\nu)\right\rangle=\int_{B_{T}} \mathrm{~d} \nu \delta H(\nu) H_{o}^{*}(\nu)=0 .
$$

The perturbation is assumed not to affect the statistical moments of field-related quantities, but only their specific random realizations. This assumption makes sense when the perturbation does not change either the complexity of the medium or the overall rate of energy dissipated. Under this assumption, introducing the average energy of $H(\nu)$, in the sense of squared $L_{2}$ norms, i.e., defined as the

$$
\mathscr{E}_{H}=\int_{B_{T}} \mathrm{~d} \nu\left\langle|H(\nu)|^{2}\right\rangle,
$$

with $\langle\cdot\rangle$ the expected value, it is straightforward to prove that

$$
\mathscr{E}_{\delta H}=\left(1-\rho^{2}\right) \mathscr{E}_{H_{o}},
$$

by enforcing the condition

$$
\mathscr{E}_{H}=\mathscr{E}_{H_{o}}
$$

and (6).

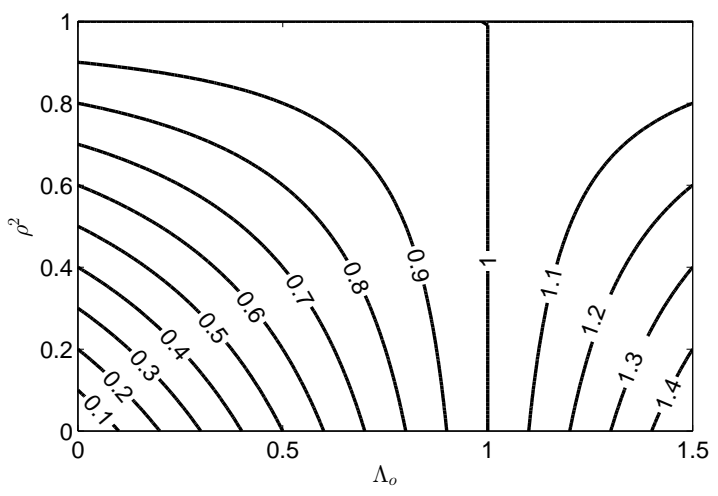

Fig. 1: $\mathscr{E}_{f} / \mathscr{E}_{f, o}$ as a function of $\rho$ and $\Lambda_{o}$.

In case of no perturbation, the Fourier spectrum of the received signal is

$$
Y_{o}(\nu)=\left|H_{o}(\nu)\right|^{2} P(\nu),
$$

while, after a perturbation occurs, one would obtain

$$
Y(\nu)=H(\nu) H_{o}^{*}(\nu) P(\nu),
$$

yielding, after (5),

$$
Y(\nu)=\rho\left|H_{o}(\nu)\right|^{2} P(\nu)+\delta H(\nu) H_{o}^{*}(\nu) P(\nu) .
$$

The degradation in the energy contrast in case of a perturbation is naturally measured by the ratio $\Lambda / \Lambda_{o}$, for which we need to compute the new energies for the coherent part of the received signal and the background fluctuations. The coherent energy $\mathscr{E}_{C}$ associated to the coherent portion of the received signal, $Y_{c}=\rho\left|H_{o}(\nu)\right|^{2} P(\nu)$, after the perturbation has occurred is given by

$$
\mathscr{E}_{c}=\rho^{2} \mathscr{E}_{c, o},
$$

by recalling (5), (6) and using the approximation (19) discussed in the Appendix.

The average energy of the background fluctuations in the perturbed case reads

$$
\mathscr{E}_{f}=\int_{B_{T}} \mathrm{~d} \nu\left|Y(\nu)-Y_{c}(\nu)\right|^{2},
$$

which can be simplified, thanks to (19), into

$$
\mathscr{E}_{f}=\rho^{2} \mathscr{E}_{f, o}+\int_{B_{T}} \mathrm{~d} \nu\left\langle|\delta H(\nu)|^{2}\right\rangle\left\langle\left|H_{o}(\nu)\right|^{2}\right\rangle .
$$

Following (8),

$$
\mathscr{E}_{f}=\rho^{2} \mathscr{E}_{f, o}+\left(1-\rho^{2}\right) \mathscr{E}_{c, o},
$$

so that

$$
\mathscr{E}_{f} / \mathscr{E}_{f, o}=\rho^{2}\left(1-\Lambda_{o}\right)+\Lambda_{o}
$$

Eq. (17) implies that the level of fluctuations in signals received in the perturbed medium should not be expected to be the same experienced in the nominal configuration. Fig. 1 shows a contour-level plot of (17); it should be noticed how, for any $\Lambda_{o} \neq 1$, perturbing the medium could either lead to weaker or stronger fluctuations, for a same loss of coherence $\rho$. 


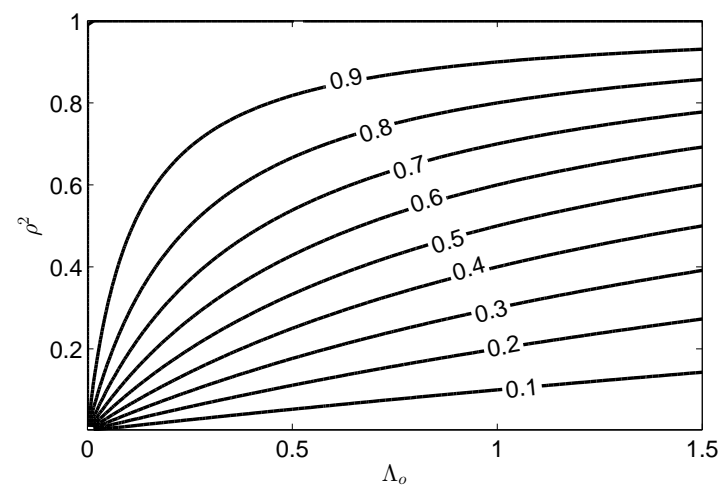

Fig. 2: $\Lambda / \Lambda_{o}$ as a function of $\rho$ and $\Lambda_{o}$.

Still, even in the case of a reduction in the fluctuations for $\Lambda_{o}<1$, the contrast systematically reduces after a perturbation, as found by combining (13) and (17)

$$
\Lambda / \Lambda_{o}=\left[1+\left(\frac{1}{\rho^{2}}-1\right) \Lambda_{o}\right]^{-1} .
$$

Fig. 2 shows how the above ratio depends on the nominal energy contrast and $\rho$. According to (17), the dependence on $\Lambda_{o}$ is purely due to the changing intensity of background fluctuations, since the received coherent energy is not expected to depend on $\Lambda_{o}$.

\section{EXPERIMENTAL VALIDATION}

In order to validate the implications of our models, access to a medium capable of supporting different field statistics is needed. A reverberation chamber is the ideal facility for these tests since, depending on the degree of overlapping in the frequency responses of its resonant modes, it can support field propagations with a varying $\Lambda_{o}$. Different values of modal overlapping, and as a consequence $\Lambda_{o}$, can be accessed by changing frequency, as demonstrated in [11], [12].

Moreover, reverberation chambers are routinely equipped with at least one metallic paddle acting as a field stirrer, which is a practical way of perturbing the medium in a controllable and reproducible way. But stirrers are usually designed and operated in order to (ideally) generate independent random realizations when rotated. This would lead to only two values of $\rho$, either 0 or 1 . We rather need to define rotation steps of the stirrer in order to evenly sample the entire range of values taken by $\rho$. As a result, the very small rotation steps used in our experiments should not come as a surprise.

Two monocone antennas were fitted as receiver and transmitter in one of CentraleSupelec's chambers, a cuboid of dimensions $6 \times 3.5 \times 2.5 \mathrm{~m}^{3}$. They were positioned on either side of the stirrer, in order to reduce the incidence of line-ofsight paths, so that modifications in the stirrer position would have an impact on the maximum number of propagation paths within the chamber.

The transfer function between the two antennas was measured with a vector network analyzer from Rohde \& Schwarz, model ZVB8, operated between $200 \mathrm{MHz}$ and $700 \mathrm{MHz}$; the transfer functions were sampled with $40 \mathrm{kHz}$ steps. This

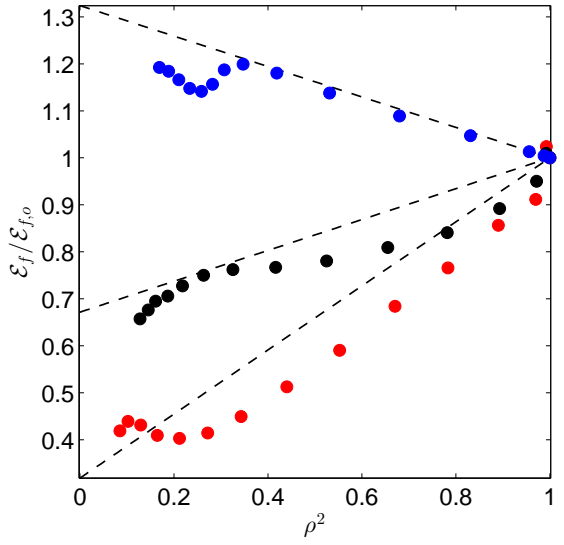

Fig. 3: Comparison between experimental (circles) and theoretical (dashed lines) relationships for the effect of a perturbation in a medium and the energy of the fluctuations at the receiver, for $\Lambda_{o}$ equal to 0.32 (red), 0.67 (black) and 1.32 (blue).

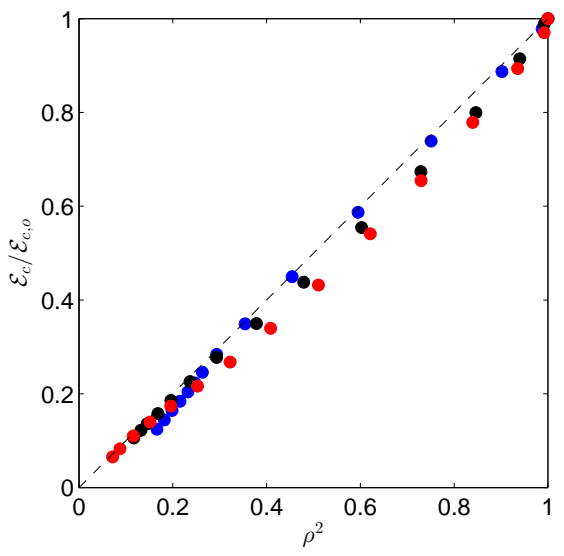

Fig. 4: As in Fig. 3, but here studying the modifications in the coherent energy at the receiver.

approach allows a sufficiently fine frequency step, in order to avoid any aliasing in the relatively long impulse responses of the chamber, around $4 \mu \mathrm{s}$.

The values of $\Lambda_{o}$ that the setup can produce were assessed from the transfer functions $H(\nu)$, as explained in [10], by estimating the normalized variance of $W(\nu)=|H(\nu)|^{2}$. Three central frequencies were picked as noteworthy, 300, 510 and $695 \mathrm{MHz}$, where the chamber behaves in a distinctly different way. A bandwidth $B_{T}=30 \mathrm{MHz}$ was chosen for the excitation around each frequency, as a compromise between spectral resolution and statistical uncertainty. For these choices, $\Lambda_{o}$ was found to be equal to $0.32,0.67$ and 1.32 , respectively, for the three central frequencies. These values correspond to the stirrer parked at its original position, in static conditions.

The stirrer was then put in motion by 1-degree steps. For each step, the new transfer function was measured, and the procedure repeated 14 times. For each frequency the linear correlation between the original transfer function and the 


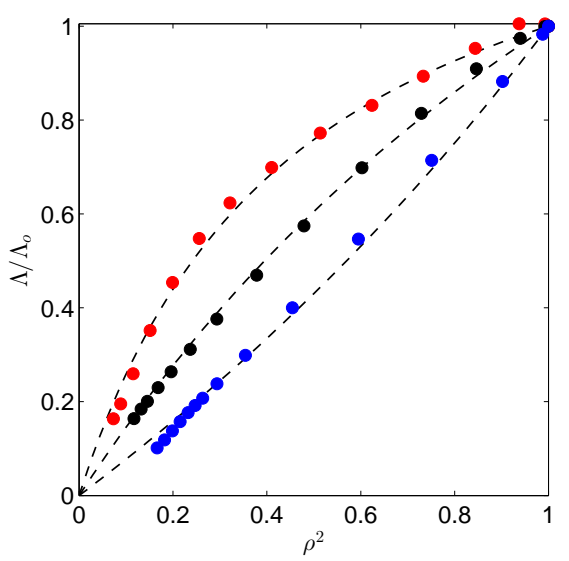

Fig. 5: As in Fig. 3, but here studying the modifications in the energy contrast at the receiver.

perturbed ones was computed, together with the resulting modification in energy contrast, coherent and backgroundfluctuation energy at the receiver. Notice that these quantities were computed by directly observing the received signals; no approximation was considered here. These results are shown in Figs. 3, 4 and 5 and compared to the theoretical models derived before.

The models appear to be accurate, as they correctly predict the strong dependence of the background-fluctuation energy on different values of the static $\Lambda_{o}$, with relative errors smaller than $20 \%$. Also, the coherent energy at the receiver is also confirmed to be practically insensitive to $\Lambda_{o}$ and proportional to $\rho^{2}$. It is therefore confirmed that the main reason for a varying robustness is the changing level of background fluctuations.

Residual errors are likely due to the use of a finite bandwidth $B_{T}$, resulting in an uncertain estimate of energy averages, which are assumed to be perfect in the model derivation. Another source of errors is the assumption of perfect homogeneity in the transfer functions of the medium as they change over time; in practice, local deviations may occur and thus infringe the assumption on which our models are based.

\section{Conclusions}

Simple theoretical models derived in this paper predict accurately how perturbations in the transfer functions of a complex medium affect TRT. The main result is the strong sensitivity of the intensity of background fluctuations to the statistics of the unperturbed medium; the coherent energy at the receiver is on the contrary unaffected by the medium statistics and directly controlled by the level of perturbation of the medium.

The accuracy of these models implies that knowing secondorder statistics of a medium TRT transfer functions and its level of potential loss of coherence are sufficient to predict the degradation in TRT schemes and related applications, e.g., imaging of moving targets or changing media.

\section{APPENDIX}

This appendix proves that given a random process $G(\nu)$ and a piece-wise smooth spectrum $P(\nu)$ of mathematical energy $\mathscr{E}_{p}$, the following approximation holds

$\int_{B_{T}} \mathrm{~d} \nu G(\nu)|P(\nu)|^{2} \simeq \int_{B_{T}} \mathrm{~d} \nu\langle G(\nu)\rangle|P(\nu)|^{2} \simeq\left\langle G\left(\nu_{c}\right)\right\rangle \mathscr{E}_{P}$,

as long as the coherence bandwidth $B_{c}^{G}$ of $G(\nu)$ is much smaller than that of $|P(\nu)|^{2}, B_{c}^{P}$. The integral can be computed over a sequence of $N$ contiguous frequency bands $B_{i}$ centered at $\nu_{i}$, covering the entire $B_{T}$, chosen such that $B_{c}^{P}>B_{i} \gg B_{c}^{G}$. In this way, $\left|P\left(\nu_{i}\right)\right|^{2}$ can be approximated as a constant over each $B_{i}$

$$
\int_{B_{T}} \mathrm{~d} \nu G(\nu)|P(\nu)|^{2} \simeq \sum_{i=1}^{N}\left|P\left(\nu_{i}\right)\right|^{2} \int_{B_{i}} \mathrm{~d} \nu G(\nu) .
$$

Each integral acts as an estimator of the ensemble average of $G\left(\nu_{i}\right)$, since $B_{i}$ spans a multitude of independent realizations, thus the first approximation in (19). In case of an average independent from frequency, the second approximation in (19) is obtained and associated to the central frequency $\nu_{c}$

\section{REFERENCES}

[1] A. Derode, A. Tourin, J. de Rosny, M. Tanter, S. Yon, and M. Fink, "Taking advantage of multiple scattering to communicate with timereversal antennas," Physical Review Letters, vol. 90, no. 1, p. 14301, 2003.

[2] R. Qiu, C. Zhou, N. Guo, and J. Zhang, "Time reversal with MISO for ultrawideband communications: Experimental results," Antennas and Wireless Propagation Letters, IEEE, vol. 5, no. 1, pp. 269-273, Dec 2006.

[3] M. Fink, "Time reversal of ultrasonic fields. I. Basic principles," IEEE Transactions on Ultrasonics, Ferroelectrics and Frequency Control, vol. 39, no. 5, pp. 555-566, 1992.

[4] H. Song, W. Hodgkiss, W. Kuperman, M. Stevenson, and T. Akal, "Improvement of time-reversal communications using adaptive channel equalizers," Oceanic Engineering, IEEE Journal of, vol. 31, no. 2, pp. 487-496, April 2006.

[5] A. Tourin, A. Derode, and M. Fink, "Sensitivity to perturbations of a time-reversed acoustic wave in a multiple scattering medium," Physical review letters, vol. 87, no. 27, p. 274301, 2001.

[6] L. Liu, D. G. Albert, and D. K. Wilson, "The effect of changing scatterer positions on acoustic time-reversal refocusing in a $2 \mathrm{D}$ urban environment at low frequencies," Journal of Geophysics and Engineering, vol. 4, no. 3, p. 276, 2007. [Online]. Available: http://stacks.iop.org/1742-2140/4/i=3/a=S06

[7] D. Liu, S. Vasudevan, J. Krolik, G. Bal, and L. Carin, "Electromagnetic time-reversal source localization in changing media: Experiment and analysis," Antennas and Propagation, IEEE Transactions on, vol. 55, no. 2, pp. 344-354, 2007.

[8] M. Yavuz and F. Teixeira, "On the sensitivity of time-reversal imaging techniques to model perturbations," Antennas and Propagation, IEEE Transactions on, vol. 56, no. 3, pp. 834-843, March 2008.

[9] B. T. Taddese, T. M. Antonsen, E. Ott, and S. M. Anlage, "Sensing small changes in a wave chaotic scattering system," Journal of Applied Physics, vol. 108, no. 11, p. 114911, 2010.

[10] A. Cozza and F. Monsef, "Multiple-source time-reversal transmissions in random media," Antennas and Propagation, IEEE Transactions on, vol. 62, no. 8, pp. 4269-4281, Aug 2014.

[11] A. Cozza, "The Role of Losses in the Definition of the Overmoded Condition for Reverberation Chambers and Their Statistics," IEEE Transactions on Electromagnetic Compatibility, no. 53, pp. 296-307, 2010.

[12] F. Monsef and A. Cozza, "Average number of significant modes excited in a mode-stirred reverberation chamber," Electromagnetic Compatibility, IEEE Transactions on, vol. 56, no. 2, pp. 259-265, 2014. 